\title{
Anastomosis ileorrectal en el tratamiento quirúrgico de la colitis ulcerosa: Resultados a largo plazo
}

\author{
Felipe Bellolio $\mathbf{R}^{1}$, José Miguel Zúñiga $A^{a}$, \\ Pablo Wagner $H^{a}$, G eorge Pinedo $M^{1}$, Ignacio D uarte $G^{2}$, \\ Álvaro Zúñiga $D^{1}$. \\ Ileorectal anastomosis in the surgical \\ treatment of ulcerative colitis: \\ Long-term results
}

Background: Total colectomy with ileorectal anastomosis (IRA) is an alternative to the ileoanal pouch for the surgical treatment of ulcerative colitis in a selected group of patients. This technique leaves rectal mucosa liable to develop persistent proctitis, dysplasia and cancer. Aim: To describe short and long-term results of IRA and to assess the presence of dysplasia. Material and methods: Descriptive study of patients treated with IRA. The data were obtained from the clinical records, and the present status was evaluated with an interview. A proctoscopy and biopsy was offered free of cost to the contacted patients. Results: Between 1978 and 2005, 26 patients were operated. One patient presented an anastomotic leakage that was treated with a loop ileostomy. There was no operative mortality. Twenty-three patients were followed for a period of 1 to 23 years. Three patients evolved as Crohn's disease and two of them needed a proctectomy. Three patients died of non-related diseases. In the remaining 17 , the average evacuation rate was $3.7 / 24 \mathrm{~h}$ and all were continent. None developed a rectal cancer. Only two patients had their planned annual endoscopic surveillance. In 2 of the 11 patients who accepted endoscopy and biopsy, a low-grade dysplasia was found. Conclusions: IRA has low morbidity and acceptable functional results in this selected group of patients. No patient present high-grade dysplasia or cancer; however, the adherence to the endoscopic follow-up is poor (Rev Méd Chile 2008; 136: 1121-6).

(Key w ords: Colitis, ulcerative; Endoscopy, digestive system; Rectal neoplasms)

Recibido el 26 de octubre, 2007. Aceptado el 5 de junio, 2008.

${ }^{1}$ Departamento de Cirugía Digestiva, ${ }^{2}$ Departamento de Anatomía Patológica, Facultad de Medicina, Hospital Clínico Pontificia Universidad Católica de Chile. Santiago, Chile aAlumno de Medicina, Pontificia Universidad Católica de Chile

Correspondencia a: Dr Álvaro Zúñiga D. Departamento de Cirugía Digestiva, Facultad de Medicina, Pontificia Universidad Católica de Chile. Marcoleta 350, Patio Interior. Santiago, Chile. Fono: 3543870. Fax: 6329620. E mail: cdigest@med.puc.cl 
$\mathrm{L}$ a colectomía total con anastomosis ileorrectal (AIR) fue impulsada por Stanley O. Aylett ${ }^{1}$ hace más de 40 años, quien comunicó buenos resultados con esta técnica en el tratamiento quirúrgico de la colitis ulcerosa (CU). Sus logros no fueron reproducidos por otros cirujanos y muchos han sido reticentes de su indicación porque los resultados funcionales son inciertos, dada la posibilidad de proctitis persistente y el riesgo de desarrollar un cáncer en la mucosa rectal crónicamente inflamada. Sin embargo, la experiencia acumulada parece todavía considerar la AIR como una alternativa actualmente válida, si se usa selectivamente ${ }^{2}$. Además, sus resultados funcionales pueden modificarse favorablemente con el uso de antiinflamatorios derivados del salicilato administrados por vía oral o rectal.

Los buenos resultados funcionales del reservorio ileal con anastomosis reservorio anal (RIA) y la eliminación de gran parte de la mucosa rectal hacen que esta técnica sea actualmente el tratamiento quirúrgico de elección para la $\mathrm{CU}^{2}$. Sin embargo, la AIR es una operación de menor complejidad, con resultados funcionales aceptables y baja morbilidad, como lo demostró este mismo grupo en una comunicación anterior ${ }^{3}$. Además, habría un menor compromiso de la fertilidad postoperatoria en mujeres comparado con el $\mathrm{RIA}^{4}$, como también menor riesgo de provocar disfunción sexual-vesical secundaria a la disección pélvica. Es así como la AIR, en un grupo seleccionado de pacientes, ha demostrado ser superior al RIA ${ }^{5-8}$, incluso describiéndose mayor dificultad técnica y morbilidad para este último ${ }^{9}$.

Dado que el RIA es actualmente el tratamiento quirúrgico de elección de la $\mathrm{CU}$, las indicaciones actuales de una AIR son restringidas y específicas para un grupo seleccionado de pacientes que presentan una o más de las siguientes condiciones: 1) diagnóstico incierto de CU, 2) pacientes reticentes a aceptar una ileostomía transitoria, 3) pacientes mayores de 60 años, 4) deseo de no comprometer la fertilidad. Así, la AIR es actualmente una indicación poco frecuente en el tratamiento quirúrgico de la CU porque presenta algunas desventajas. Dado que se conserva la mucosa rectal enferma tienen un riesgo potencial de reactivar la enfermedad en el muñón rectal o desarrollar un cáncer del recto a largo plazo. Por lo tanto, el seguimiento prolongado en este grupo de pacientes es de importancia en la evaluación de esta alternativa quirúrgica. El compromiso de seguimiento endoscópico del recto remanente es un requisito fundamental para la indicación operatoria.

El objetivo de esta comunicación es describir los resultados quirúrgicos y funcionales a largo plazo de la AIR en pacientes con CU y evaluar el estado actual de la mucosa rectal remanente con estudio endoscópico e histológico.

\section{PACIENTES Y MÉTODO}

En este estudio descriptivo se identificó, en la base de datos de pacientes operados por enfermedad inflamatoria intestinal, a todos los pacientes sometidos a colectomía total con anastomosis ileorrectal por CU entre enero 1978 y diciembre 2005. Se consignaron datos demográficos y variables de la evolución clínica pre y postoperatoria. El diagnóstico de CU se hizo basado en el cuadro clínico, estudio endoscópico e histopatológico de las biopsias endoscópicas y mediante el estudio anatomopatológico de las piezas operatorias.

Los pacientes en los cuales se consideró indicada una AIR se comprobó que cumplieran los siguientes criterios:

- indicación operatoria electiva,

- parámetros nutricionales normales,

- ausencia de displasia en la mucosa rectal,

- distensibilidad rectal conservada y continencia anal normal, ambas evaluadas clínicamente,

- suspensión de terapia esteroidal al menos 30 días antes de la AIR, y

- aceptación por parte del paciente de biopsias rectales anuales para seguimiento.

En los pacientes sometidos en primera instancia a una colectomía total de urgencia y conservación del muñón rectal, se esperó un lapso mínimo de 4 meses antes de realizar la AIR. En los pacientes sometidos a colectomía y AIR en la misma operación la colectomía se practicó en forma clásica o por vía laparoscópica. Se seccionó el íleon inmediato a la válvula ileocecal y el colon en la unión recto sigmoidea a nivel del promontorio, de manera de dejar un muñón rectal de aproximadamente $15 \mathrm{~cm}$. En los pacientes con una colectomía previa, se desmanteló la ileosto- 
mía y se preparó el muñón rectal de manera de seccionarlo a nivel del promontorio. La anastomosis ileorrectal fue realizada en todos los pacientes en forma látero-terminal, con sutura manual o mecánica.

Se realizó una encuesta a los pacientes evaluando su calidad de vida actual en cuanto a frecuencia de evacuaciones en $24 \mathrm{~h}$, continencia y uso de medicamento específicos por vía oral o rectal. También se les invitó a realizarse un control médico sin costo con rectoscopia y biopsias múltiples de la mucosa rectal remanente, previo consentimiento informado. Las muestras histológicas fueron analizadas e informadas por el mismo grupo de patólogos.

\section{Resultados}

En el período estudiado, 26 pacientes fueron sometidos a AIR. Catorce $(53,8 \%)$ de estos pacientes eran mujeres, con una edad promedio de 38 años (i: 11-68). Un paciente fue operado por vía laparoscópica. Tres pacientes (11\%) presentaron complicaciones postoperatorias: uno $(3,8 \%)$ presentó una fístula de la anastomosis que requirió una ileostomía transitoria, otro paciente tuvo una

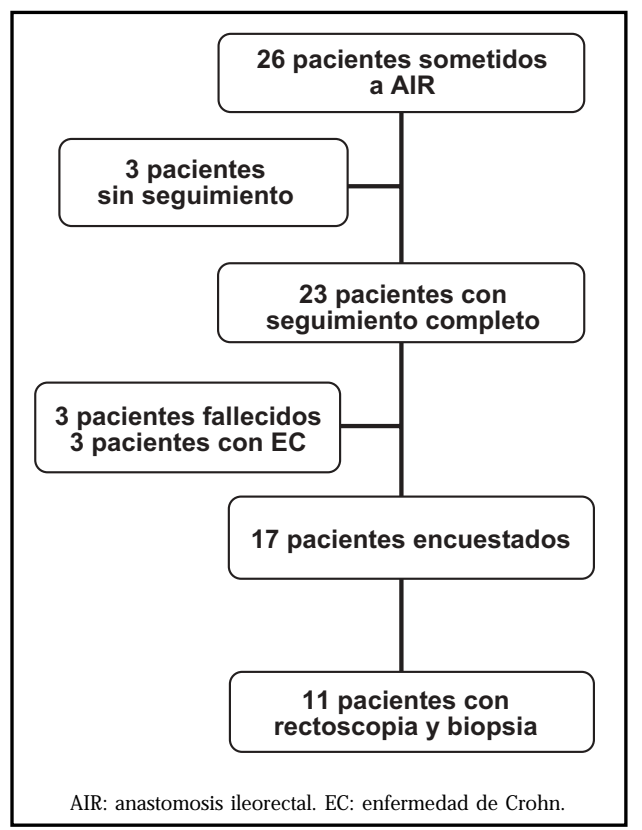

Figura 1. Organigrama de flujo de los pacientes del estudio. infección de la herida operatoria y el tercero desarrolló un absceso perianal. No hubo mortalidad operatoria.

Se logró seguimiento completo en 23 (88\%) de los 26 pacientes, con un promedio de seguimiento de 144 meses (intervalo 12-340) (Figura 1). De los 23 pacientes con seguimiento completo, tres (13\%) habían fallecido por causas no relacionadas con la CU ni con la AIR. Otros tres (13\%) evolucionaron en el largo plazo como una enfermedad de Crohn con compromiso rectal y perineal.

En los 17 pacientes restantes (edad promedio 34 años, 10 mujeres), la evaluación funcional demostró que 13 (76\%) tenían 4 o menos evacuaciones en $24 \mathrm{~h}$, con un promedio de evacuaciones diarias de 3,7 (rango: 1-6). Nueve de los encuestados presentaba al menos un episodio de deposiciones nocturnas (promedio 1,6, rango 1-4). Un solo paciente $(5,8 \%)$ refería incontinencia ocasional a gases. Seis (35\%) recibían algún tipo de tratamiento con derivados del aminosalicilato (tres reciben 5-ASA orales y tres con 5-ASA tópico) en el control y tres de ellos utilizaban algún antidiarreico intermitentemente. Ninguno había desarrollado cáncer del recto ni tampoco la necesidad de proctectomía por reactivación de la CU. Siete eran mujeres con edades entre 11 y 40 años, observándose que cinco lograron embarazarse después de la AIR. Un paciente de este grupo había presentado episodios de obstrucción intestinal que no habían requerido cirugía, y ninguno había presentado estenosis anastomótica.

En cuanto al seguimiento endoscópico, sólo dos (12\%) de los 17 pacientes cumplían regularmente con la recomendación de una rectoscopia anual y biopsias rectales. Los 15 pacientes restantes llevaban un control clínico irregular que habitualmente no habían incluido rectoscopias ni biopsias.

Once de los 17 pacientes con CU aceptaron realizarse un control con rectoscopia y biopsias múltiples (Tabla 1). Los pacientes que accedieron al control endoscópico tenían un seguimiento post quirúrgico entre 7 y 28 años, y nueve (81\%) de ellos con seguimiento superior a diez años. En 10 pacientes la rectoscopia demostró mucosa de aspecto granular, con dibujo vascular visible pero atenuado, sin signos inflamatorios y fue catalogado como proctitis inactiva. Sólo un paciente 
Tabla 1. Pacientes con colitis ulcerosa en seguimiento y control endoscópico e histopatológico

\begin{tabular}{|cccc|}
\hline Paciente & $\begin{array}{c}\text { Años de } \\
\text { seguimiento }\end{array}$ & Hallazgo endoscópico & Hallazgo histológico \\
\hline 1 & 8 & Proctitis ulcerosa inactiva & sin displasia \\
2 & 24 & Proctitis ulcerosa inactiva & colitis crónica activa, sin displasia \\
3 & 21 & Proctitis ulcerosa inactiva & sin displasia, colitis inespecífica leve \\
4 & 21 & Proctitis ulcerosa inactiva & sin displasia \\
5 & 7 & Proctitis ulcerosa inactiva & sin displasia \\
6 & 16 & Proctitis ulcerosa inactiva & sin displasia \\
7 & 28 & Proctitis ulcerosa inactiva & sin displasia, colitis crónica \\
8 & 24 & Proctitis ulcerosa inactiva & displasia de bajo grado, \\
& & colitis ulcerosa inactiva & sin displasia \\
9 & 22 & Proctitis ulcerosa inactiva & displasia de bajo grado \\
10 & 15 & Proctitis ulcerosa leve & colitis crónica activa \\
11 & 13 & Proctitis ulcerosa inactiva & sin displasia, colitis crónica activa \\
\hline
\end{tabular}

presentó actividad inflamatoria leve y en ninguno se encontró lesiones perianales (fístula/fisura). En el estudio histopatológico de las biopsias rectales no se encontró carcinoma y sólo dos informan displasia epitelial de bajo grado, una de las cuales además presenta proctitis crónica activa.

\section{Discusión}

El análisis de los resultados de esta serie muestra que los pacientes sometidos a colectomía total y AIR por CU presentan buenos resultados funcionales en cuanto a frecuencia de deposiciones en $24 \mathrm{~h}$, continencia y necesidad de medicación, tal como se describe en el análisis de series similares (Tabla 2). Efectivamente, la frecuencia promedio de evacuaciones (3,7/día) y la mantención de continencia observada en este grupo de pacientes es similar a la descrita por otros autores ${ }^{6,10}$. Esto confirma que los resultados funcionales de la AIR dependerán de una adecuada selección de los pacientes, en los que la conservación de la distensibilidad del recto, la ausencia de actividad inflamatoria, la continencia y la ausencia de displasia en la mucosa rectal son fundamentales.

El principal inconveniente de la AIR es la preservación de la mucosa rectal que puede dar origen a reactivación de la enfermedad y eventual- mente a largo plazo un cáncer del recto. En un metaanálisis reciente se estimó que el riesgo acumulativo de desarrollar cáncer en un paciente con CU es de $2 \%$ a 10 años, $8 \%$ a 20 años y $18 \%$ a 30 años ${ }^{11}$. En nuestra serie, pese a la búsqueda dirigida que se realizó mediante rectoscopias y biopsias rectales, no se demostró adenocarcinoma rectal en ninguno de los pacientes. Sólo dos estudios histopatológicos en pacientes con seguimiento mayor a 10 años presentaron displasia epitelial leve, uno de ellos con actividad inflamatoria. Es importante destacar que la presencia de displasia en el contexto de inflamación pierde su valor como marcador de riesgo de desarrollo de neoplasia, por lo que se hace necesario descartar que los cambios encontrados regresen con el uso de tratamiento específico. Al comparar nuestros resultados con lo descrito en otras series, encontramos que la frecuencia de cáncer de recto en pacientes sometidos a una AIR es baja, alcanzando aproximadamente 5\% (Tabla 2). Sin embargo, cabe destacar que las series publicadas son en general de pocos pacientes, con tiempos de seguimiento variable. Esta baja frecuencia de cáncer rectal hace posible plantearse la opción de realizar una AIR en un grupo seleccionado de pacientes con CU, previo compromiso del paciente de mantener controles endoscópicos regulares para la pesquisa oportuna de lesiones premalignas y malignas de la 


\section{Tabla 2. Series publicadas de pacientes con colitis ulcerosa sometidos a una colectomía y anastomosis ileorrectal}

\begin{tabular}{|lccl|}
\hline Autor (Referencia) & $\mathrm{N}^{\circ}$ pacientes & Años de seguimiento & Frecuencia cáncer rectal o LP \\
\hline Pastore $^{7}$ & 48 & $6,5$ (i: $0-19)$ & $2 \%$ carcinoma \\
& & & $2 \%$ displasia \\
Baker $^{14}$ & 374 & Hasta 23 años & $5,9 \%$ carcinoma \\
Khubchandani $^{17}$ & 68 & $22(\mathrm{i}: 1-30)$ & $2,9 \%$ carcinoma invasor \\
& & & $4,4 \%$ displasia (1 CIS) \\
Leijonmarck & & 13 (i: $4-27)$ & $0 \%$ carcinoma \\
& 51 & $14,2$ (i: $1-28)$ & $0 \%$ displasias \\
Serie UC & 26 & & $7,7 \%$ displasia \\
& & & \\
\hline
\end{tabular}

LP: Lesión premaligna. CIS: Carcinoma in situ.

mucosa rectal. Sin embargo, pese a la insistencia de la importancia de esta medida, el seguimiento endoscópico en este grupo es deficiente, ya que sólo 2 de 17 pacientes mantuvieron controles regulares. Aparentemente, los buenos resultados funcionales de la operación y la calidad de vida con escasas limitaciones hacen que los pacientes no consideren necesario un control endoscópico.

En el largo plazo, tres (13\%) de los 23 pacientes en seguimiento evolucionaron finalmente como una $\mathrm{EC}$, dos de los cuales requirieron de una proctectomía. Leijonmark y cols, en una serie de 60 pacientes sometidos a AIR por CU, encontraron que nueve (15\%) fueron diagnosticados como EC al revisar las biopsias ${ }^{12}$. A pesar que el diagnóstico fue postoperatorio, en este grupo de enfermos el beneficio del procedimiento es mayor, ya que se evita realizar un RIA en un paciente con alta posibilidad de fracaso por compromiso del reservorio o por desarrollo de enfermedad perianal.

En nuestra serie, ninguno de los 17 pacientes con CU requirió de proctectomía en su seguimiento, lográndose el control de las reactivaciones inflamatorias con tratamiento médico, a diferencia de lo observado en la literatura, en que se describe que hasta $13 \%$ de los pacientes necesitarán una proctectomía por reactivación de la enfermedad $^{9,13}$. Esto se puede explicar porque la serie es pequeña o por una adecuada selección de los pacientes a los que se les ofrece la AIR.
Otra característica que hace atractiva a la AIR es su simplicidad técnica y baja morbilidad. Nuestra serie presentó 3,8\% de falla en la anastomosis, lo cual es comparable con otras series que presentan tasas de filtración de hasta $6 \%^{9,12}$. También se describe un menor compromiso vesical y disfunción sexual comparada con el RIA, dado que la AIR no requiere de una disección pélvica muy extensa. Es así como la AIR tendría una mejor preservación de la fertilidad en mujeres con deseo de embarazarse, lo cual se puede observar en nuestra serie de pacientes en que 5 de las 7 mujeres operadas en edad fértil logró embarazarse. Por lo anterior, debe considerarse el deseo de embarazo como una de las variables a tomar en cuenta al analizar las alternativas del tratamiento quirúrgico en estos pacientes particularmente si se toma en cuenta la disminución de la fertilidad en mujeres en edad fértil sometidas a RIA $^{14,15}$.

Pese a los beneficios y buenos resultados funcionales que ofrece la AIR, el RIA seguirá siendo el tratamiento de elección para la mayoría de los pacientes portadores de CU, dado que en esta última no hay preservación de mucosa rectal evitando la posibilidad de reactivar la enfermedad o de desarrollar un cáncer rectal. Sin embargo es importante considerar que el RIA presenta mayor morbilidad y resultados funcionales inferiores que la AIR, tomando en consideración las diferencias entre los pacientes que son sometidos a una y otra 
técnica. En la serie de RIA de la Cleveland Clinic, la mayor publicada hasta el momento con 1.005 pacientes, 521 de los cuales eran por CU, se observó un promedio de 6 evacuaciones diarias, $29 \%$ de pacientes con algún grado de incontinencia y sobre $50 \%$ de complicaciones postoperatorias $^{16}$. Además, los pacientes con RIA están en riesgo de desarrollar una reservoritis crónica, la que se observa hasta en $30 \%$ de los pacientes con RIA por $\mathrm{CU}^{2}$.

En resumen, se puede afirmar que la AIR en un grupo de pacientes seleccionado tiene plena indicación. En el seguimiento a largo plazo de estos pacientes encontramos muy buenos resultados funcionales y bajo riesgo de desarrollar cáncer de la mucosa rectal remanente lo cual pudimos

\section{REFERENCIAS}

1. AyLETT SO. Three Hundred Cases of Diffuse Ulcerative Colitis Treated by Total Colectomy and IleoRectal Anastomosis. BMJ 1966; 1: 1001-5.

2. Blumberg D, Beck D. Surgery for ulcerative colitis. Gastroenterol Clin N Am 2002; 31: 219-35.

3. ZúNítga A, Rivera C, López F, Rahmer A, Cortés P. Rol actual de la anastomosis ileorrectal en el tratamiento quirúrgico de la colitis ulcerosa. Rev Chil Cir 1994; 46: 535-9.

4. Mortier PE, Gambiez L, Karoui M, Cortot A, Paris JC, QuandaLe P ET AL. Colectomy with ileorectal anastomosis preserves female fertility in ulcerative colitis. Gastroenterol Clin Biol 2006; 30: 594-7.

5. Hawley P. Ileorectal anastomosis. Br J Surg (Suppl) 1985; 75-82.

6. Oakiey J, Jagelman DG, Fazio VW, Lavery IC. Complications and quality of life after ileorectal anastomosis for ulcerative colitis. Am J Surg 1985; 149: 108-14.

7. Schoetz DJ, CoLer JA, Veidenheimer MC. Alternative to conventional ileostomy in chronic ulcerative colitis. Surg Clin N A 1985; 65: 21-33.

8. FAZIO VW. The role of ileorectal anastomosis for ulcerative colitis. En: Allan RN (ed), Inflamatory Bowel Diseases, Londres, Churchill Livingstone 1990; 429-38.

9. Pastore RLO, Wolff BG, Hodge D. Total abdominal colectomy and ileorectal anastomosis for inflammatory bowel disease. Dis Colon Rectum 1997; 40: 1455-64. confirmarlo mediante control endoscópico y biopsias. La baja frecuencia de cáncer presente en el muñón rectal a largo plazo hace posible plantear la AIR como opción de tratamiento para la CU, siempre y cuando el paciente se comprometa a mantener controles anuales con rectoscopia y biopsias. No hay estudios que, basados en datos objetivos, recomienden justificadamente un control anual. Respecto a este punto, es nuestra opinión que éste debe ajustarse al tiempo de evolución de la CU previo a la AIR y a los hallazgos de la mucosa rectal después del primer examen. Así se puede disminuir el impacto que causa en los pacientes una rectoscopia anual, que se suma a los múltiples estudios endoscópicos del colon que el paciente sufrió previo a la AIR.
10. NEWTON CR, BAKER WN. Comparison of bowel function after ileorectal anastomosis for ulcerative colitis and colonic polyposis. Gut 1975; 16: 785-91.

11. EAden JA, Abrams KR, MayberRy JF. The risk of colorectal cancer in ulcerative colitis: a meta-analysis. Gut 2001; 48: 526-35.

12. Leijonmark CE, Löfberg R, Öst A, Heliers G. Long term results of ileorectal anastomosis in ulcerative colitis in Stockholm County. Dis Colon Rectum 1990; 33: 195-200.

13. Khubchandani IT, Kontostolis SB. Outcome of ileorectal anastomosis in an Inflammatory bowel disease surgery. Experience of three decades. Arch Surg 1994; 129: 866-9.

14. Gorgun E, Remzi FH, Goldberg JM, Thornton J, Bast J, HUL TL ET AL. Fertility is reduced after restorative proctocolectomy with ileal pouch anal anastomosis: a study of 300 patients. Surgery 2004; 136: 795-803.

15. Johnson P, Richard C, Ravid A, Spencer L, Pinto E, HanNa M ET AL. Female infertility after ileal pouchanal anastomosis for ulcerative colitis. Dis Colon Rectum 2004; 47: 1119-26.

16. Fazio VW, Ziv Y, Church JM, Oakiey JR, Lavery IC, MILSOM JW ET AL. Ileal pouch-anal anastomosis complications and function in 1005 patients. Ann Surg 1995; 222: 120-8.

17. BaKer WN, Giass RE, Ritchie JK, Aylett SO. Cancer of the rectum following colectomy and ileorectal anastomosis for ulcerative colitis. Br J Surg 1978; 65: 862-8. 